\section{Resident recognition and treatment of hypercholesterolaemic patients}

Sir,

Effective treatment of hypercholesterolaemia reduces the risk of coronary heart disease (CHD). Even after the National Institutes of Health (NIH) Consensus Conference of 1985, studies reported that hypercholesterolaemia was recognized in only $47 \%$ to $66 \%$ of in-patients. ${ }^{1-4}$ In 1988 , a nationwide physician education program was initiated by the release of the National Cholesterol Education Program Committee (NCEP) guidelines. ${ }^{5}$ One study suggests that family practitioners' recognition of hypercholesterolaemia has increased since then. ${ }^{6}$ However, the effect of the guidelines on resident physician recognition and treatment of hypercholesterolaemia is unknown. This study was performed to assess the frequency of recognition and treatment of hypercholesterolaemia by resident physicians in the inpatient setting after the publication of the NCEP guidelines.

Records of all admissions to the medical service at the Medical College of Georgia (MCG) from August 1988 to February 1989 were screened. Patients whose cholesterol was not measured, who died during the admission, or whose chart was unavailable for review were excluded. Other exclusion criteria were admission to the intensive care unit or from the emergency room, dialysis, and diagnosis of malignancy or HIV disease. Charts of the 320 patients not excluded were reviewed. Laboratory printouts were examined to determine if serum cholesterol was greater than $240 \mathrm{mg} / \mathrm{dl}(6.24 \mathrm{mmol} / \mathrm{l})$. Twenty-five per cent (50) of 203 general medicine patients and $40 \%$ (47) cardiac catheterization patients had an elevated serum cholesterol. These 97 patients comprised the study group. Their charts were reviewed to determine if elevated cholesterol was recognized and if a diagnostic or therapeutic action was undertaken.

Patients were then classified as general medicine or cardiac catheterization admissions. The age, sex, serum cholesterol level, and prior history of hyperlipidaemia or coronary heart disease were recorded. The number of risk factors, including male sex, diabetes mellitus, hypertension, family history of CHD, tobacco use, and obesity was tallied. Chi-square analysis between the groups was computed for frequency of recognition, diagnostic and therapeutic actions, sex and history of hyperlipidaemia. Student's $t$-tests were computed between the groups for mean differences in age, cholesterol level, and number of risk factors.

Sixty per cent of the hypercholesterolaemic patients were recognized and $57 \%$ underwent a diagnostic or therapeutic action. Comparisons between general medicine and cardiac catheterization patients for mean age, cholesterol level, male sex, and history of hyperlipidaemia or CHD were nonsignificant. General medicine patients had significantly fewer risk factors than cardiac catheterization patients $(2.2$ vs $2.7, P<0.05)$. Hypercholesterolaemia was recognized in $38 \%$ of patients admitted to general medicine services and $83 \%$ of those admitted for cardiac catheterization $(P<0.01)$. No action was undertaken in $66 \%$ of general medicine and $19 \%$ of cardiac catheterization patients $(P<0.01)$ Cholesterol lowering diet was ordered significantly more often for cardiac catheterization patients.

We conclude that many residents often do not recognize or treat hypercholesterolaemia in the in-patien setting at the Medical College of Georgia despite publication and dissemination of the NCEP guidelines. Hypercholesterolaemia is recognized and treated significantly more often if the patient is admitted for cardiac catheterization than to a general medicine service.

\section{Ruth-Marie E. Fincher, Laura Q. Rogers, Lori M. Herring, ${ }^{2}$ Lloyd A. Lewis, Leonard D. Nance, III, 'Department of Medicine ${ }^{2}$ Department of Surgery \\ ${ }^{3}$ The Curriculum Office, Medical College of Georgia, Augusta, Georgia, ${ }^{4}$ Department of Medicine University of Tennessee School of Medicine, Memphis} Tennessee, USA

\section{References}

1. Consensus Conference: Lowering blood cholesterol to prevent heart disease. JAMA 1985, 253: 2080-2086.

2. Madlon-Kay, D.J. Family physician recognition and treatment of severe hypercholesterolemia. J Fam Pract 1987, 24: $54-56$.

3. Bell, M.M. \& Dippe, S.E. Recognition and treatment of hypercholesterolemia in a family practice center. J Fam Pract 1988, 26: 507-513.

4. Landzberg, J.S. \& Heim, C.R. Physician recognition and treatment of hypercholesterolemia. Arch Intern Med 1989, 149: 933-935.

5. The Expert Panel: Report of the National Cholesterol Education Program Expert Panel on detection, evaluation and treatment of high blood cholesterol in adults. Arch Intern Med 1988, 148: 36-61.

6. Madlon-Kay, D.J. Improvement in family physician recognition and treatment of hypercholesterolemia. Arch Intern Med 1989, 149: 1754-1755.

\section{Spontaneous hepatic rupture due to metastatic malignant melanoma}

Sir,

The liver is a common site for the growth of metastatic deposits of malignant melanoma. The following case report demonstrates a rare complication of this condition. 